\section{GRP-183 THE EFFECT OF A CLINICAL PHARMACIST-LED TRAINING PROGRAMME ON INTRAVENOUS PREPARATION AND ADMINISTRATION ERRORS IN A VIETNAMESE HOSPITAL}

doi:10.1136/ejhpharm-2013-000276.183

${ }^{1} \mathrm{HT}$ Nguyen, ${ }^{2} \mathrm{TD}$ Nguyen, ${ }^{3} \mathrm{ER}$ van den Heuvel, ${ }^{4} \mathrm{FM}$ Haaijer-Ruskamp, ${ }^{1} \mathrm{~K}$ Taxis. ${ }^{1}$ University of Groningen, Pharmacotherapy \& Pharmaceutical Care, Groningen, The Netherlands; 2University of Medicine and Pharmacy at Ho Chi Minh city, Clinical Pharmacy, Ho Chi Minh, Vietnam; 'University Medical Center Groningen, Epidemiology, Groningen, The Netherlands; ${ }^{4}$ University Medical Center Groningen, Clinical Pharmacology, Groningen The Netherlands

Background Medication safety has been a concern for decades worldwide, but there is still relatively little research about interventions to reduce medicines administration errors in hospitals, especially in resource-restricted settings such as Vietnam. Our large study on the frequency and type of medication errors in Vietnamese hospitals indicated that the highest risk was associated with intravenous medication administration [1]

Purpose To investigate the effect of intensive training on the frequency of intravenous medicines preparation and administration errors in an urban public hospital in Vietnam.

Materials and Methods This was a controlled intervention study with pre- and post-intervention measurements using a direct observation method, carried out in two critical care units: Intensive Care Unit (ICU - intervention ward), and Post-Surgical Unit (PSU control ward). The intervention consisted of lectures plus practical ward-based teaching sessions, carried out by a clinical pharmacist and a nurse. In each ward, all intravenous doses prepared and administered by nurses were observed 12 hours per day, on 7 consecutive days, each period.

Results A total of 1294 doses were observed, 718 in ICU and 576 in PSU. Error rate on the intervention ward (ICU) decreased from $62.7 \%$ to $52.5 \%$ ( $\mathrm{P}=0.01$ ); preparation errors including wrong dose, deteriorated drug, wrong technique of preparation decreased significantly $(p<0.05)$. On the control ward (PSU) there was no significant change in error rates $(73.8 \%$ vs. $73.1 \%, p=0.85)$; almost all preparation error types were similar in both periods $(p>0.05)$, except for technique errors, which was increased from $15.5 \%$ to $25.9 \%(p<0.05)$.

Conclusions Intensive training showed a slight improvement in overall and specific error rates, particularly preparation errors. Further measures are needed to improve patient safety.

Reference

1. EAHP abstract titled: "Errors in medication preparation and administration in Vietnamese hospitals", by H.T. Nguyen et al,

No conflict of interest.

\section{GRP-184 THE IMPACT OF PHARMACEUTICAL INTERVENTIONS ON THE TREATMENT OF GRAM POSITIVE INFECTIONS}

doi:10.1136/ejhpharm-2013-000276.184

A Sendra García, 0 Ruiz Millo, P Campillos Alonso, M Climente Martí. Hospital Universitario Dr. Peset, Pharmacy, Valencia, Spain

Background Inappropriate use of antibiotics results in increased antibiotic resistance and poor efficiency, which should be avoided through pharmaceutical interventions.

Purpose To evaluate the impact of pharmaceutical interventions (PIs) on the effectiveness, safety and efficiency of treatment of Gram-positive infections in adult in-patients

Materials and Methods For 4 months, all episodes of hospitalisation (on the same Gram positive antibiotic treatment) were evaluated of adult in-patients who were on vancomycin, linezolid or daptomycin for $\geq 24$ hours, except for the indication of prophylaxis.
Variables related to: 1) Patient [sex, age, penicillin allergy or intolerance, hospitalisation unit ( $\mathrm{HU})$ and type of setting-acquired infection, diagnosis, length of stay], 2) treatment duration, drug and observance of the criteria of use established by the drug therapeutics committee (DTC), considering treatment of choice (vancomycin) and alternative treatments (linezolid and daptomycin), and 3) PIs: number, type (effectiveness, safety or efficiency), pharmacotherapeutic medication process, drug, type of PI (discontinuation of medicine, suggested therapeutic alternative, initiation of the medicine, dose individualization (DI), therapeutic/clinical drug monitoring (TCDM) and acceptance of the PI.

SPSS v. 17.0 was used.

Results 148 patients [(59\% male; mean age 67 years $(95 \% \mathrm{CI}$ : 63-68) and penicillin allergy/intolerance: 10\%] received 174 treatments. $76 \%$ patients were on medical HU; the infection originated in the community (85\%); Diagnosis: bacteraemia (23\%), skin and soft tissues infection (21\%), pneumonia (20\%). Median duration of hospital stay: 16 days (IOR: 9-27); of antibiotic treatment: 7 days (IOR: 3-11).

Most prescribed antibiotic: vancomycin (68\%) [linezolid (28\%), daptomycin (3\%)]. 74\% (128) of treatments fulfilled criteria established by the DTC; linezolid and vancomycin didn't fulfil the criteria in $35 / 49(71 \%)$ and $9 / 118(8 \%)$ prescriptions.

251 PIs were made, 96 (38\%) during initial prescription validation, representing $1 \mathrm{PI} /$ treatment (IOR: $1-2$ ) and generating 79\% acceptance. Type of intervention: safety $44 \%$ (93\% in vancomycin), effectiveness $24 \%$ (94\% in vancomycin) and efficiency $32 \%$ (83\% in linezolid). After the PI, 84\% (146) treatments met DTC criteria, the percentage of non-conforming linezolid decreasing to $23 / 49(47 \%)$ 155 PIs (IOR: 1-3) were performed during follow-up, with 2PIs/ treatment and an 87\% acceptance; these were mainly DI (48\%) and TCDM (42\%) interventions.

Conclusions Pharmaceutical interventions in patients with Grampositive infections increase treatment efficiency and pursue improvement of the effectiveness and safety throughout the antibiotic treatment, reflecting the need for continued treatment followup to adapt it to the patient's clinical course.

No conflict of interest.

\section{GRP-185 THE PHARMACIST'S ROLE IN EMERGENCY FIRST AID SERVICES IN A TERRORIST ATTACK WITH SARIN: EMERGENCY INTERVENTION SIMULATION}

doi:10.1136/ejhpharm-2013-000276.185

${ }^{1}$ A Cecchi, ${ }^{2}$ Carchietti. 'University Hospital Udine, Pharmacy, Udine, Italy; ${ }^{2}$ University Hospital Udine, Director EMS and regional HEMS, Udine, Italy

Background After 11 September 2011, Italy prepared a Public Health Plan for national defence and regional storage facilities for antidotes. These are managed by a physician and a pharmacist. In Friuli Venezia Giulia-Italy, the pharmacist is responsible for the safety of the antidotes, the national database, collaborates with the physician in planning for emergencies and makes antidotes available for immediate transfer to the site of the incident. Sarin, a nerve gas, even at a very low concentration, causes death rapidly if the victim isn't treated immediately with atropine and subsequently within the first 4-5 hours with pralidoxime.

Purpose To verify, by means of a simulation, that there were sufficient stocks of atropine, and the accessibility, distribution and the appropriateness of the treatment.

Materials and Methods We simulated a terrorist attack with sarin at the railway station in Udine, the seriousness equivalent to the attack in Tokyo on 20 March 1995.

Results In Tokyo, 107 people out of approximately 6000 involved in the attack with sarin, needed treatment with atropine. $80 \%$ were treated with only $2 \mathrm{mg}$, for a total of $170 \mathrm{mg}$, while 21 needed more 
than $2 \mathrm{mg}$. Nobody was given more than $9 \mathrm{mg}$. In total, $350 \mathrm{mg}$ of atropine was immediately necessary on the site of the attack, equivalent to 350 phials of $1 \mathrm{mg}$. In our simulation, the time for access and preparation of the antidote was about 10 minutes from the moment of the alert. The transfer and distribution time to the site was less than 15 minutes due to favourable road access, geographical factors and the short distance from the station to the storage facility.

Conclusions The pharmacist is responsible for immediate availability, accessibility and distribution of the antidotes to the site of emergency, and awareness of appropriate treatment.

No conflict of interest.

\section{GRP-186 THE QUALITY OF ORAL CHEMOTHERAPY PRESCRIBING IN ONCOHAEMATOLOGIAL OUTPATIENTS}

doi:10.1136/ejhpharm-2013-000276.186

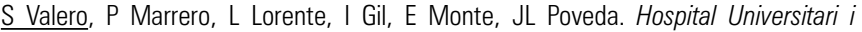
Politecnic La Fe, Hospital Pharmacy, Valencia, Spain

Background Nowadays, in our health area, most of the oral antineoplastic drugs prescribed to outpatients are dispensed in hospital pharmacy services. Patients receiving these kinds of drugs are susceptible to suffering adverse events (AE) due to medicines errors (MEs). Purpose To evaluate the quality of oral chemotherapy drug prescriptions (OCDPs) in oncohaematological outpatients.

Materials and Methods Descriptive prospective study. OCDPs for adult patients received in a pharmaceutical outpatient care unit were analysed for two months. The information necessary for OCDPs was established based on legal rules and international recommendations. We established that omitted or confused information in patient identification (identification number), weight, height and/or corporal surface (in drugs dosed depending on these parameters), diagnosis, treatment duration, dose and frequency of administration, presented serious risk based on possible consequences.

Results 291 prescriptions were analysed from 183 patients. 100\% of prescriptions had almost one omission, $78.7 \%$ of which showed serious errors of omitted or confused information related to the following items: patient identification $(0.7 \%)$, weight, height or corporal surface $(56.7 \%)$, diagnosis $(28.9 \%)$, treatment duration $(14.1 \%)$, dose $(5.8 \%)$ or frequency $(12.1 \%)$. Information omitted or confused about patient and treatment information included: age or birth date $(1.4 \%)$, allergies (omitted $56 \%$, unknown $38.8 \%$ ), morbidities $(59.5 \%)$, cycle number $(67 \%)$ and periodicity $(46.7 \%)$. Drug information omitted or confused included: drug name (generic $35.7 \%$, originator $61.5 \%$ or both $2.7 \%$ ), dose units $(10.7 \%)$, pharmaceutical form $(83.1 \%)$ or route of administration $(58.4 \%)$. Physician information omitted or confused included: name $(7.6 \%)$, signature $(1 \%)$ and collegiate number (1\%).

Conclusions Our results show a high rate of omitted and confused information in prescriptions in OCDP. Extreme attention during the validation process was required in order to prevent MEs and AEs. New tools, such as electronic prescription, pre-printed medical orders or educational programmes for prescribers, must be implemented in order to improve the quality of OCDP.

No conflict of interest.

\section{GRP-187 THE RATES AND TYPES OF PRESCRIBING ERRORS IN ELECTRONIC CHEMOTHERAPY PRESCRIPTIONS FOR AMBULATORY PATIENTS}

doi:10.1136/ejhpharm-2013-000276.187

'M Dobravc Verbic, ${ }^{2} \mathrm{~K}$ Kantilal, ${ }^{3} \mathrm{~N}$ Barber. ' UNIVERSITY MEDICAL CENTRE LJUBLJANA, Pharmacy, Ljubljana, Slovenia; 'Guy's and St Thomas' NHS Foundation Trust, Pharmacy, London, UK; ' UCL School of Pharmacy, Department of Practice and Policy, London, UK
Background Electronic prescribing (EP) systems have been recognised as successful in reducing chemotherapy prescribing errors. However, electronic prescriptions are unlikely to prevent all errors, and new types of errors may emerge.

Purpose To assess prescribing error rates and identify new error types and their causes with the implementation of a electronic prescribing system for ambulatory cancer patients at a London Cancer Centre.

Materials and Methods A service evaluation was conducted in two parts, covering two different strategies for interception of prescribing errors - prospectively by pharmacists during a 2 -week period, and retrospectively using data from the pharmacy EP telephone helpline service, over 41 weeks.

Results The overall rate of error-containing prescriptions was estimated to be $6 \%$.

In the prospective part, 32 errors were identified from 571 electronic chemotherapy prescriptions. Most commonly committed errors were chemotherapy drug dose adjustments (13; 41\%) and weight omissions (11; 34\%).

In the retrospective analysis, 95 of 141 errors (67\%) were 'e-selection errors', classified mainly as 'work-arounds' (26; 18\%), 'wrong commands' (35; 25\%), or 'wrong fields' (27; 19\%). 63 errors (45\%) were related to scheduling a chemotherapy or supportive drug or regimen.

Electronic system-related causes of prescribing errors were recognised in 4 of 32 cases (13\%) in the prospective part, and in 89 of 141 cases $(63 \%)$ in the retrospective part. It was estimated that with implementation of technical solutions and additional prescriber training, $85 \%$ of these errors could be prevented in the future.

Conclusions The estimated rate of chemotherapy prescribing errors was $6 \%$. A number of different errors, specific for electronic prescribing, were identified, with a thorough explanation of how various errors may have occurred. Future larger scale studies are needed to confirm prescribing error rates, and to possibly identify other, previously unrecognised, types of chemotherapy prescribing errors.

No conflict of interest.

\section{GRP-188 UNDER-REPORTING OF ADVERSE DRUG REACTIONS IN THE HOSPITAL SETTING: AN ESTIMATE BASED ON THE ANALYSIS OF HOSPITAL DISCHARGE RECORDS}

doi:10.1136/ejhpharm-2013-000276.188

'MC Leo, 'E Cini, ${ }^{2} \mathrm{~A}$ Pugi, 'R Banfi. 'Careggi University Hospital, Pharmacy, Florence, Italy; ${ }^{2}$ University of Florence, Centre of Pharmacovigilance Department of Pharmacology, Florence, Italy

Background In the post-marketing setting, spontaneous reporting is an important tool for the surveillance of Adverse Drug Reactions (ADRs). However, underreporting is a major limitation of a pharmacovigilance system. Several studies showed that ADRs may cause hospitalisation resulting in an increase in hospital stays and costs.

Purpose To gather information on the extent and frequency of ADRs at Careggi University Hospital, and to identify unreported ADRs to the Pharmacovigilance Office, using the hospital discharge records.

Materials and Methods We analysed the hospital discharge records from January 2011 to June 2012. In particular, we considered those records with a Drug Related Group (DRG) classification related to allergic reactions, poisoning and toxic effects of drugs (DRGs from 447 to 451). We included in our analysis records referring to poisoning, according to the new pharmacovigilance legislation in force from July 2012. Our research gave us information about the number of suspected reactions, but it didn't provide specific information on the patients and the seriousness of the reaction. 